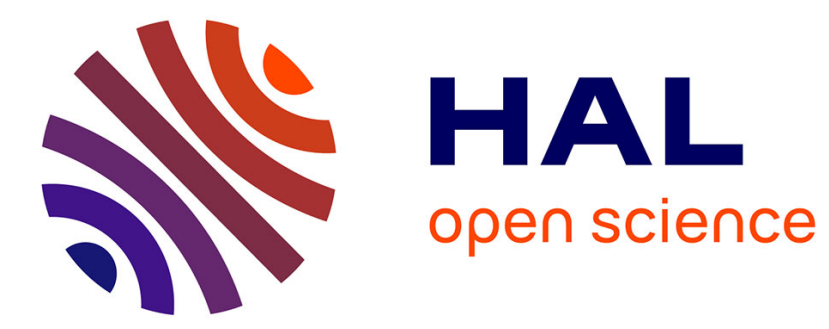

\title{
A necessary condition for weak lumpability in finite Markov processes \\ James Ledoux
}

\section{To cite this version:}

James Ledoux. A necessary condition for weak lumpability in finite Markov processes. Operations Research Letters, 1993, 13 (3), pp.165-168. 10.1016/0167-6377(93)90006-3 . hal-00852842

\section{HAL Id: hal-00852842 \\ https://hal.science/hal-00852842}

Submitted on 3 Sep 2013

HAL is a multi-disciplinary open access archive for the deposit and dissemination of scientific research documents, whether they are published or not. The documents may come from teaching and research institutions in France or abroad, or from public or private research centers.
L'archive ouverte pluridisciplinaire HAL, est destinée au dépôt et à la diffusion de documents scientifiques de niveau recherche, publiés ou non, émanant des établissements d'enseignement et de recherche français ou étrangers, des laboratoires publics ou privés. 


\title{
A Necessary Condition \\ for Weak Lumpability in Finite Markov Processes
}

\author{
James Ledoux* \\ IRISA - INRIA \\ Campus de Beaulieu, F-35042 Rennes Cedex, FRANCE
}

26 November 1992

\begin{abstract}
Under certain conditions, the state space of a homogeneous Markov process can be partitionned to construct an aggregated markovian process. However, the verification of these conditions requires expensive computations. In this note, we expose a necessary condition for having a markovian aggregated process. This condition is based on properties of the eigenvalues of certain submatrices of the transition rate matrix of the original Markov process.
\end{abstract}

Subject classification: Probability: Markov processes, Markov chains, Lumpability.

${ }^{*}$ This work was partially supported by the grant 290C2010031305061 of the French Region Bretagne. 


\section{Introduction}

Markov processes are frequently used as analytic models in quantitative evaluations of systems. In the particular case of computer systems, they are useful in the resolution of performance or reliability problems. In a wide class of situations, the user does not need informations about each state of its model, but about state classes only. This leads to considering a new process, called the aggregated process, whose states are the state classes of the original one. To keep all the power of Markov processes theory, it is important to be able to claim that, for a given initial distribution, the aggregated process is still markovian. This property is called weak lumpability (see Kemeny [3]). The strong lumpability is the particular case where any initial distribution leads to an aggregated markovian process. Necessary and sufficient conditions have been studied in [3], Rubino and Sericola [6],[7] for irreducible processes. These conditions are very expensive to verify: in the worst case, the cost grows exponentially with the number of states [6].

A related problem analysed in Barr and Thomas [1] leads to necessary conditions for strong lumpability of Markov chains. In spite of an easy extension to continuous time processes, their work can not be applied to the more general problem of weak lumpability. In this general situation, we give a new necessary condition based on the eigenvalues of the submatrices composed of the transition rates between states of the same class only.

In Section 2, we review briefly the definition and caracterization of the weak and strong lumpability properties of a finite Markov process. In Section 3, we recall the expression of the distributions of sojourn times in a subset of states of a Markov process. Section 4 is 
devoted to the new necessary condition for weak lumpability. We show in Section 5 how to benefit by the result of Section 4 for making easier the search of partitions allowing the use of the aggregated process instead of the original one.

\section{Weak and strong lumpability of Markov processes}

Let $\left(X_{t}\right)_{t \geq 0}$ be a homogeneous irreducible Markov process, $E=\{1, \cdots, N\}$ be its state space and $A$ be its transition rate matrix. This process $X$ is completly specified by its initial probability distribution $\alpha$ on $E$. It will be denoted by $(\alpha, A)$. We denote by $\pi$ the associated stationary distribution (i.e. the unique probability vector satisfying $\pi A=0$ ). Consider the partition $\mathcal{P}=\{C(1), \cdots, C(M)\}$ of the state space in $M$ classes, $M<N$. To the given process $X$, we associate the aggregated stochastic process $Y$, denoted by $\operatorname{agg}(\alpha, A, \mathcal{P})$, with values on $F=\{1, \ldots, M\}$ and defined by

$$
Y_{t}=m \Longleftrightarrow X_{t} \in C(m)
$$

Following [7], a necessary and sufficient condition for having the weak lumpability of $X=(\alpha, A)$ with respect to $\mathcal{P}$, i.e. for having an aggregated markovian process, is that $\alpha$ must satisfy a linear system with $N \times(M+1)^{N}$ equations; its expression is not important here. Furthermore, the following property holds.

Theorem 2.1 [7] When $X$ is weakly lumpable there exists an unique matrix $M_{\pi}$, independent of $\alpha$, so that the aggregated Markov process admits as infinitesimal generator

$$
\widehat{A}=M_{\pi} A B
$$


where $\forall i \in E, \forall j \in F, B(i, j)=1$ if $i \in C(j)$ and 0 otherwise and $\forall i \in F, M_{\pi}(i, j)=$ $\pi(j) / \sum_{k \in C(i)} \pi(k), \forall j \in C(i)$ and 0 otherwise.

Example (adapted from [3]). Let $A$ be the transition rate matrix

$$
\left(\begin{array}{c|cc}
-18 & 6 & 12 \\
\hline 0 & -20 & 20 \\
21 & 3 & -24
\end{array}\right) .
$$

Consider the partition $\mathcal{P}=\{C(1), C(2)\}$ with $C(1)=\{1\}$ and $C(2)=\{2,3\}$. The stationary distribution is $\pi=(7 / 16,3 / 16,3 / 8)$. The matrices $B$ and $M_{\pi}$ are respectively

$$
B=\left(\begin{array}{cc}
1 & 0 \\
0 & 1 \\
0 & 1
\end{array}\right), \quad M_{\pi}=\left(\begin{array}{ccc}
1 & 0 & 0 \\
0 & \frac{1}{3} & \frac{2}{3}
\end{array}\right) .
$$

We get from (2)

$$
\widehat{A}=\left(\begin{array}{cc}
-18 & 18 \\
14 & -14
\end{array}\right)
$$

The strongly lumpable particular case Barr and Thomas [1] dealt with, is the situation in which every initial probability distribution on $E$ yields to a markovian aggregated process. The necessary and sufficient condition is that $A$ satisfies the equation

$$
B M A B=A B
$$

where $M=\left(B^{t} B\right)^{-1} B^{t}$ is independent of $\pi ;()^{t}$ denotes the transpose operator. In this case we have $\widehat{A}=M A B$. 
Remark. These results are valid in discrete time, substituting matrix $A$ by the transition probability matrix $P$ (see [3],[7]). The first theorem in [1] states that, if the Markov chain is strongly lumpable, then the eigenvalues of the probability transition matrix $\widehat{P}$ of $\operatorname{agg}(\alpha, A, \mathcal{P})$ (a partition $\mathcal{P}$ is fixed) are also eigenvalues of $P$. We can easily extend this property to Markov processes and to weak lumpability. This is not the case with the second theorem of [1] in the weak lumpability situation, essentially because the relation (3) is only a sufficient condition for weak lumpability ([3], page 136). Hence we can not use their procedure to point out "candidate" lumpings of $E$.

\section{Sojourn times in a subset of states}

Let $D$ be a subset of the space state $E$. Rubino and Sericola [5] have shown that, for $n \geq 1$, the random variable $S_{D, n}$ "time spent during the $n^{\text {th }}$ sojourn of $X$ in $D$ " has the distribution

$$
\mathbb{P}_{\alpha}\left\{S_{D, n} \leq t\right\}=1-u_{n}(\alpha) e^{A_{D} t} 1^{T}
$$

where $u_{n}(\alpha)$ is the probability distribution of the $D$-valued random variable "state from which the $n^{\text {th }}$ sojourn of $X$ in $D$ begins" and $A_{D}$ is the submatrix of $A$ consisting of the transition rates between the states of $D$ only.

If the process $\operatorname{agg}(\alpha, A, \mathcal{P})$ is markovian, sojourn times in a given state $m \in F$ are exponentially distributed with parameter $\widehat{A}(m, m)$. This implies that the right hand side of (4) involving process $X$ is reduced to $1-\exp (\widehat{A}(m, m) t)$, for $D=C(m)$. The study of this possible reduction will lead to the main result of this note. 


\section{A necessary condition}

The necessary condition for the process $\operatorname{agg}(\alpha, A, \mathcal{P})$ to be markovian is obvious from Section 3. The relation (4) must be reduced to $\mathbb{P}_{\alpha}\left\{S_{C(m), n} \leq t\right\}=1-\exp (\widehat{A}(m, m) t)$, for all $C(m) \in \mathcal{P}$. In fact, we shall prove that, for every state $m \in F, \widehat{A}(m, m)$ must be an eigenvalue of the matrix $A_{C(m)}$.

Theorem 4.1 Let $\mathcal{P}$ be a given partition of the state space $E$ and let $\widehat{A}=M_{\pi} A B$. If $\operatorname{agg}(\alpha, A, \mathcal{P})$ is markovian then $\widehat{A}(m, m)$ is an eigenvalue of the matrix $A_{C(m)}$ for each $m \in F$.

Proof. If $\operatorname{agg}(\alpha, A, \mathcal{P})$ is markovian, we have for each $n \geq 1$ and $t \geq 0$

$$
u_{n}(\alpha) e^{A_{C(m)} t} 1^{T}=e^{\widehat{A}(m, m) t}
$$

In the sequel, we omit the parameters $n$ et $\alpha$ in the notation because they are not essential to the discussion.

The matrix $A_{C(m)}$ is similar to a diagonal matrix of $p$ Jordan's blocks, each of them being associated with an eigenvalue $\lambda_{k}$ of $A_{C(m)}$ distinct of the remaining $p-1$ eigenvalues (Ortega [4], page 181). The previous relation can be expressed in the following form:

$$
\forall t \geq 0, \quad \sum_{k=1}^{l} e^{\lambda_{k} t} P_{k}(t)=e^{\widehat{A}(m, m) t}
$$

where $l \leq p$, the $\lambda_{k}$ 's are each to each distincts and the $P_{k}(t)$ are non-zero polynomials.

We recall that any finite set of functions of the form $t^{i} \exp \left(\lambda_{k} t\right)$, where the $i$ 's are nonnegative integers and the $\lambda_{k}$ 's are complex numbers, is linearly independent on any 
nonvoid open interval, unless two or more functions are identical (see e.g. Birkhoff and Rota [2], page 79).

If we rewrite (6) as

$$
\forall t \geq 0, \quad \sum_{k=1}^{l} e^{\lambda_{k} t} P_{k}(t)-e^{\widehat{A}(m, m) t}=0
$$

we deduce, applying the previous recalled result, that there exists an unique integer $k_{0}$ such that

$$
\lambda_{k_{0}}=\widehat{A}(m, m) \text { and } P_{k_{0}}(t) \equiv 1 \text {. }
$$

Remark. If the matrix $A_{C(m)}$ is irreducible we have the more precise result

$$
\widehat{A}(m, m)=\rho_{1}
$$

where $\rho_{1}$ is the real eigenvalue of the matrix $A_{C(m)}$ such that $\rho_{1}>\operatorname{Re}\left(\lambda_{k}\right)$, for all the remaining eigenvalues $\lambda_{k}$. This is based on the fact that (see Seneta [8], page 47):

$$
e^{A_{C(m)} t}=e^{\rho_{1} t} V+O\left(e^{\tau t}\right)
$$

where $\tau<\rho_{1}$ and where the real matrix $V$ is positive. Then the polynomial associated with $\rho_{1}$ is non-zero and therefore $\rho_{1}$ appears in the list of the $\lambda_{k}$ 's in (6).

The theorem also holds for an irreducible Markov chain. The expression of the sojourn time distribution in a state subset $D$ is, following [5],

$$
\forall q \geq 1, \quad \mathbb{P}_{\alpha}\left\{S_{D, n}=q\right\}=u_{n}(\alpha) P_{D}^{q-1}\left(I d-P_{D}\right) 1^{T}
$$


where $u_{n}(\alpha)$ et $P_{D}$ have the same meaning than in (4). Then, for each classe $C(m)$, the distribution in (7) must be reduced to a geometric distribution with parameter $\widehat{P}(m, m)$. A similar proof, with Jordan reduction of $P_{C(m)}$, leads to the following result.

Corollary 4.2 Let $\mathcal{P}$ be a given partition of the state space $E, P$ be the transition probability matrix and let $\widehat{P}=M_{\pi} P B$. If agg $(\alpha, P, \mathcal{P})$ is markovian then $\widehat{P}(m, m)$ is an eigenvalue of the matrix $P_{C(m)}$ for each $m \in F$.

Proof. The main formula (6) becomes in discrete time

$$
\forall q \geq 1, \quad \sum_{k=1}^{l} \lambda_{k}^{q-1}\left(1-\lambda_{k}\right) P_{k}=\widehat{P}(m, m)^{q-1}(1-\widehat{P}(m, m))
$$

where the $P_{k}$ are polynomials in $q$. As in the proof of Theorem 4.1 , we recall that a finite set of functions of the form $q^{i} \lambda_{k}{ }^{q}, i$ being a nonnegative integer and $\lambda_{k}$ a complex number, is linearly independent, unless two or more functions are identical. This implies that there exists an integer $k_{0}$ such that $\lambda_{k_{0}}=\widehat{P}(m, m)$ and $P_{k_{0}}=1$.

Example. With the same matrix $A$ as in the previous example, we can prove that for $\alpha=(0,1 / 3,2 / 3)$ the process $\operatorname{agg}(\alpha, A)$ is markovian. It is easy to verify that $\widehat{A}(2,2)=$ -14 is an eigenvalue of

$$
A_{C(2)}=\left(\begin{array}{cc}
-20 & 20 \\
3 & -24
\end{array}\right) \text {. }
$$




\section{Conclusion}

We must emphasize that weak lumpability is a desirable property for evaluating the transient behaviour of Markov processes. It is clear that computing $\widehat{A}$ (or $\widehat{P}$ ) implies explicit knowledge of the stationary probability vector $\pi$ associated with $A$ (or $P$ ). Hence, this takes classical "aggregation" methods for obtaining stationary probability distribution of a Markov process apart. This being stated, we can use this necessary condition to obtain "candidate" lumpings of $E$ leading to an aggregated markovian process, as in [1]. This condition allows the preselection of "candidate" partitions before applying the characterization of weak lumpability in terms of the solution to some linear systems [6]. We propose here the following procedure. The first step consists of determining the stationary vector $\pi$ associated with $A$. For each partition $\mathcal{P}$, we calculate $\{\widehat{A}(m, m), m \in$ $F\}$ with

$$
\widehat{A}(m, m)=\frac{\sum_{i \in C(m)} \sum_{j \in C(m)} \pi(i) A(i, j)}{\sum_{i \in C(m)} \pi(i)}
$$

and we verify that, for each $m \in F, \widehat{A}(m, m)$ is an eigenvalue of $A_{C(m)}$. In this case, $\mathcal{P}$ is a potential partition of $E$ leading to an aggregated markovian process. Contrarily to [1], we are sure that actual lumping partitions leading to aggregated markovian processes are detected by the procedure above. 


\section{References}

[1] Barr D.R. and M.U. Thomas. "An Eigenvector Condition for Markov Chain Lumpability". Operations Res. 25,1029-1031 (1977).

[2] Birkhoff G. and Rota G-C. Ordinary Differential Equation. John Wiley \& Sons, 1989.

[3] Kemeny J.G. and J.L. Snell. Finite Markov Chains. Springer-Verlag, New York, 1976.

[4] Ortega J.M. Matrix Theory: a second course. Plenum Press, New York, 1987.

[5] Rubino G. and B. Sericola. "Sojourn Times in Finite Markov Processes". J. Appl. Prob. 27,744-756 (1989).

[6] Rubino G. and B. Sericola. "A Finite Caracterization of Weak Lumpable Markov Chains Part I". Stoch. Proc. and Appl., 38, 195-204 (1991).

[7] Rubino G. and B. Sericola. "A Finite Caracterization of Weak Lumpable Markov Processes Part II : The continuous time case." Stoch. Proc. and Appl., 45, 115-125, (1993).

[8] Seneta E. Non-Negative Matrices and Markov Chains. Springer-Verlag, New York, 1981. 